\title{
Medicina di Precisione e Medicina di Laboratorio
}

\author{
Precision Medicine and Laboratory Medicine
}

\author{
Piero Cappelletti ${ }^{1}$ (I)
}

Ricevuto: 4 settembre 2016 / Accettato: 9 settembre 2016 / Pubblicato online: 3 ottobre 2016

(C) Società Italiana di Patologia Clinica e Medicina di Laboratorio 2016

Riassunto Nonostante qualche incertezza semantica derivante dalla sovrapposizione con i concetti di Medicina Personalizzata, la Medicina di Precisione (Precision Medicine) è intesa come "un approccio emergente di trattamento e prevenzione delle malattie che tiene conto della variabilità individuale di geni, ambiente e stili di vita di ciascuna persona". Gli obiettivi sono focalizzati a breve termine sul cancro e a lungo termine verso conoscenze globali applicabili a tutto l'ambito della salute e malattia. Entrambe si basano sulle recenti scoperte molecolari e bioinformatiche, sono potenziate dal convergere di tecnologie che consentono una sempre maggiore connettività, anche attraverso i social media e i mobile device, richiedono un ripensamento della filosofia di ricerca e sono condizionate dall'empowerment dei pazienti/cittadini come partner nella ricerca medica e nel mantenimento di salute individuale e collettiva. Naturalmente vi sono barriere, legate ai costi e alla competizione per i fondi, alla preparazione dei medici ma in generale degli stakeholders e a critiche concettuali di metodo, di obiettivo e di filosofia sanitaria. Tuttavia la Medicina di Precisione sta imponendosi, spinta da iniziative come quella del Presidente Obama, ed è una opportunità interessante per la Medicina di Laboratorio, poiché a condurre l'approccio terapeutico è largamente la diagnostica (Precision Diagnostics) che richiede "qualità" metodologica, una comprensione corretta del rapporto tra innovazione ed "evidenze" che caratterizza la Medicina Traslazionale e nuovi ruoli per la Medicina di Laboratorio come la Patologia Terapeutica.

Parole chiave Medicina di Precisione - Medicina

Personalizzata Genomica $\cdot$ Big data $\cdot$ Medicina

Traslazionale $\cdot$ Medicina di Laboratorio

$凶$ P. Cappelletti

pie.cappelletti@gmail.com

1 SIPMeL, Castelfranco Veneto, TV, Italia
Summary Although some confusion exists with terms such as Personalized Medicine or 4Ps Medicine, Precision Medicine is defined as "an emerging approach for disease treatment and prevention that takes into account individual variability in genes, environment, and lifestyle for each person". The US Precision Medicine Initiative has both short-term and long-term goals: expanding in the area of cancer research and then to all areas of health and healthcare on a large scale. These goals are based on molecular and bioinformatics developments, converging technologies of connectivity, new models of biomedical research, and the empowerment of patients and citizens as partners in healthcare research and organization. Of course, some barriers derive from the costs and the competition for funding, preparedness of doctors and stakeholders, and criticism about medical methodology, targets, and philosophy of Precision Medicine. Nevertheless, the Precision Medicine is growing and is an outstanding opportunity for Laboratory Medicine because a new Precision Diagnostics should guide the targeted therapies. For this role, methodological quality, correct understanding of the relationship between innovation and evidence building, and new skills such as Therapeutic Pathology are essential.

Keywords Precision Medicine Personalized Medicine . Genomics · Big data $\cdot$ Translational Medicine $\cdot$ Laboratory Medicine

\section{Introduzione}

Il termine Precision Medicine (Medicina di Precisione, MP) è divenuto di conoscenza comune dopo l'annuncio del Presidente Obama il 25 gennaio 2015 sulla Precision Medicine Initiative [1], che dedicava 215 milioni di dollari del budget 
presidenziale 2016 al fine di "accelerare le scoperte biomediche e fornire ai clinici nuovi strumenti, conoscenze e terapie per selezionare i trattamenti che funzionano meglio per ciascun paziente", seguito l'anno successivo dall'iniziativa Moonshot to Cure Cancer. Tuttavia, le basi concettuali della MP in Oncologia affondano in un paio di decadi precedenti e la sua prima formale definizione è fatta risalire al 2009 per opera di Mark Boguski [2], Co-Fondatore e Direttore Medico del Precision Medicine Network e Professore alla Harvard Medical School dove ha fondato la Genomic Medicine Initiative.

La Precision Medicine Initiative molto probabilmente darà risultati importanti solo a lungo termine, ma cresce sempre più lo stimolo a precorrere i tempi, anche in Paesi diversi dagli USA come UK [3] e Canada [4]. In Italia se ne è parlato a proposito dell' Human Technopole Italy 2040 da insediare nell' area Expo di Milano [5].

\section{Definizione di Medicina di Precisione}

Secondo il National Institutes of Health (NIH) [6], la MP è "un approccio emergente di trattamento e prevenzione delle malattie che tiene conto della variabilità individuale di geni, ambiente e stili di vita di ciascuna persona". Tale approccio dovrebbe consentire ai medici e ai ricercatori di predire più accuratamente quale trattamento e/o prevenzione per una specifica malattia funzionerà in un certo gruppo di pazienti e/o di persone. È un concetto molto diverso da quello "one-size-fits-all" normalmente praticato e focalizzato alle strategie terapeutiche e preventive per il paziente "medio". Il termine è stato formalmente discusso e accettato dal $\mathrm{Na}$ tional Research Council of the National Academies (NRC) nel 2011 [7].

Secondo Boguski [2], la MP ha tre essenziali attributi: una comprensione deterministica dell'eziologia e patogenesi delle malattie; la capacità di diagnosticare gli specifici fattori causali e l'abilità di trattare specificamente ed efficacemente le cause profonde della malattia. Non si tratta di concetti nuovissimi, ma la cui possibilità di applicazione è drammaticamente aumentata per lo sviluppo di database biologici di larga scala, come il sequenziamento del genoma umano, di metodi potenti di caratterizzazione dei pazienti, come le "omiche", le analisi cellulari e la tecnologia "mobile", e di nuovi strumenti computazionali, capaci di analizzare grandi serie di dati [8].

Come si può notare, vi sono sovrapposizioni tra i termini "Medicina di Precisione" e "Medicina Personalizzata" oppure, ancora, con "medicina 4P" (predittiva, preventiva, personalizzata, partecipativa) [9]. Secondo il NRC, "Personalized Medicine" è un termine più antico ma dal significato similare a "Precision Medicine" e, tuttavia, osserva che il termine "personalizzato" potrebbe essere mal interpretato come rivolto esclusivamente al singolo paziente, mentre il termine "preciso" dovrebbe chiarire che il focus è identificare quale approccio sarà efficace per quali pazienti sulla base dei fattori genetici, ambientali e di stile di vita. Per tali ragioni il NRC preferisce il termine "Precision Medicine" [10]. Secondo altre visioni [11], mentre il termine "Precision Medicine" è strettamente legato alla genomica e alla sua capacità di evidenziare le costellazioni genomiche caratteristiche delle malattie che costituiscono target specifici di terapie mirate (farmacogenomica), il concetto di "Personalized Medicine", che incorpora quello di 4Ps Medicine, sarebbe più omnicomprensivo e puntato alla costruzione di modelli adatti alle malattie degenerative croniche, sulla pianificazione preventiva della cura piuttosto che sulla terapeutica reattiva al fatto acuto, coinvolgendo cittadini e pazienti.

Tuttavia, se è ovvio che il termine MP ha a che fare con la Genomica e la Farmacogenomica [12], secondo il NCR fa un passo avanti perché non si definisce più in un concetto statico. Piuttosto [13] ha l'obiettivo di affrontare il punto saliente dell'ambiguità del quadro clinico e dell'incertezza della cura, attraverso l'integrazione dei vari livelli o strati di conoscenza del soggetto in una visione complessiva ("panoromica" direbbe Topol [14]), accostata per far comprendere il concetto-in "Toward Precision Medicine: Building a Knowledge Network for Biomedical Research and a New Taxonomy of Disease" [10] del NRC-alla costruzione del GIS (geographic information system) delle mappe Google. In questi termini MP è strettamente collegata alla System Medicine o Medicina della Complessità [15].

Di fatto, però, in letteratura la maggior parte degli autori utilizza i termini in modo intercambiabile, anche se un dibattito approfondito, come quello sul termine "Personalised Medicine", sarebbe necessario [16].

\section{Obiettivi e benefici attesi}

Il NCR offre esaustive informazioni sulla Precision Medicine Initiative e sul ruolo che a essa è assegnato dalle aspettative istituzionali e accademiche [17]. Gli obiettivi sono focalizzati a breve termine sul cancro e a lungo termine verso conoscenze globali applicabili a tutto l'ambito della salute e malattia. Entrambe si basano sulle recenti scoperte molecolari e bioinformatiche [8], sono potenziate dal convergere di tecnologie che consentono una sempre maggiore connettività [18], anche attraverso i social media e i mobile device, richiedono un ripensamento della filosofia di ricerca [13] e sono condizionate dall'empowerment dei pazienti/cittadini come partner nella ricerca medica e nel mantenimento di salute individuale e collettiva [8].

L'attenzione sul cancro, primario tema sanitario dell'Occidente, dovrebbe consentire di superare alcuni degli ostacoli incontrati dalla Precision Oncology: resistenze ai farmaci 
inspiegate, eterogeneità genomica dei tumori, monitoraggio della risposta terapeutica e delle ricadute più sensibile e tempestiva e migliore conoscenza delle interazioni tra farmaci, integrando e migliorando l'attuale schema interpretativo. Le conoscenze metodologiche relative alla ricerca, alle nuove tassonomie e all'uso combinato dell'armamentario terapeutico/preventivo dovrebbero essere la base per un' analoga MP in altri campi, come quelli già sperimentati delle malattie psichiatriche [19] o di quelle allergiche [20].

I benefici, almeno potenziali, attesi dalla Precision Medicine Iniziative sono relativi a una migliore protezione dei dati dei pazienti, all'utilizzo di nuovi strumenti per l'analisi dei big data, alla definizione degli standard FDA per lo sviluppo di test e terapie, a nuove partnership inter-specialistiche e coinvolgenti pazienti e organizzazioni sanitarie e a opportunità per milioni di persone di partecipare all'avanzamento della conoscenza. A più lungo termine, ci si attende che: l'utilizzo delle informazioni genetiche e molecolari divenga parte integrante della routine medica; la capacità predittiva dei trattamenti specifici faccia un balzo in avanti; la comprensione dei meccanismi patogenetici delle malattie migliori anche attraverso una nuova tassonomia; la migliore integrazione dei dati digitalizzati (EHR, electronic health record; h-GIS, human geographic information system) consenta accessi veloci e sicuri dei dati globali e strutturati; l'approccio preventivo, diagnostico e terapeutico di molte malattie migliori; infine, che ciò comporti un migliore utilizzo e un minore costo dell'organizzazione sanitaria [7].

\section{Problemi, barriere e resistenze}

La tensione ad accelerare l'introduzione di strumenti di MP sollecita problemi, barriere e resistenze [21]. Le novità della ricerca molecolare e genomica e ancor più quelle più globali della MP pongono nuove sfide. I produttori di farmaci sono chiamati ad accelerare il flusso dell'innovazione; devono modificare il loro approccio i finanziatori pubblici (nuovi fondi e nuove strategie) e privati (le Assicurazioni devono rivedere le regole per garantire i trattamenti specifici); i pazienti affrontano conoscenze e costi sanitari che crescono esponenzialmente; i medici curanti devono velocemente diventare esperti di nuovi campi per garantire la migliore stratificazione diagnostica e prognostica e, di conseguenza, la migliore terapia ai propri assistiti [22]. I medici e i ricercatori devono modificare il proprio modo di affrontare la ricerca clinica muovendo dai classici clinical trail basati sulle caratteristiche topografiche e istologiche del tumore agli umbrella o basket trail dove la casistica è raggruppata in base alle caratteristiche molecolari insieme con l'istotipo o dalle sole caratteristiche molecolari del genotipo. Si lavora su piccoli numeri con modellizzazioni complesse. Ma il problema principale è la pratica clinica: per esempio, gli oncologi medici dovrebbero applicare schemi terapeutici che rompono le tradizionali compartimentazioni organizzative legate a organi e tessuti [22]. D'altra parte l'utilizzo dei big data facilita la comprensione globale della vulnerabilità, ma richiede metodi di studio e applicativi concettualmente diversi dagli schemi deterministici e ipotetico-deduttivi comunemente conosciuti [13].

Le principali barriere [22] riconosciute alla penetrazione della MP sono: il costo economico di per sé, i collegati timori che le risorse convogliate su di essa vadano a discapito di altre aree sanitarie estremamente sensibili come quella della prevenzione e lo scetticismo sull'effettiva de-intensificazione dei trattamenti conseguenti a una migliore caratterizzazione molecolare del tumore e del paziente/gruppo di pazienti specifico; la resistenza al cambiamento di pratiche assistenziali e schemi terapeutici consolidati, che coinvolge la preparedness dei medici e del personale sanitario nei confronti dell'innovazione di MP. Da quest'ultimo punto di vista, i risultati in diverse parti del mondo non sono molto differenti: in Italia un'indagine [23] di "Recenti Progressi in Medicina" ha dimostrato una diffusa disinformazione se non impreparazione, cosi come le inchieste in ambito USA [24]. È importante però comprendere le diverse accezioni del termine "preparati", identificando le entrustable professional activities (EPA) che comprendono "le attività professionali che nel loro insieme costituiscono la massa di elementi critici che operativamente definiscono una professione" per poter costruire gli strumenti educativi più opportuni [25].

Ma le resistenze più profonde sono quelle concettuali legate a uno scetticismo di fondo, che vede la proposta come non realistica o addirittura fuorviante. Alcuni [26] ricordano che la MP non è la creazione di farmaci individuali, ma di terapie per gruppi identificati da mutazioni specifiche, che la penetranza di specifiche alterazioni genetiche è condizionata dagli eventi epigenetici che poco conosciamo e da quelli ambientali che invece possiedono un impatto granitico, che abbiamo evidenze sull'efficacia di alterazioni specifiche nella tassonomia ma non sull'efficacia, in particolare a lungo termine, delle terapie correlate e concludono che il cammino è ancora lungo, complicato e costoso e richiede cautela. Altri [27] apertamente ricordano che, nel produrre il carico di malattie degenerative croniche, sono molto più importanti i determinanti sociali (fattori di rischio, stili di vita ecc.) che minime alterazioni genetiche o epigenetiche in piccoli gruppi o sottogruppi di pazienti e che quindi quelli dovrebbero essere il target di grandi sforzi istituzionali. Per questi autori la MP rischia di essere un' arma di "distrazione" di massa. Infine, la MP è vista come contraddittoria rispetto alla strutturazione sanitaria impostata sui PDTA (clinical pathway) standardizzati e basati sull'evidence-based medicine delle migliori pratiche clinicamente provate [22]. La risposta [28] è che i due livelli dovrebbero muoversi contestualmente a un livello macro (cura delle popolazioni) e a 
uno micro (individuale) e che la chiave per consentire un reciproco beneficio e una riduzione dei costi è la condivisione dell'informazione tra paziente, fornitore di servizi, pianificatori e gestori della sanità pubblica, garantita esclusivamente dall'interoperabilità dei sistemi digitali secondo $i$ criteri HIMSS [29].

\section{Precision diagnostics}

Il faro di MP è puntato sulle terapie più efficaci e meno tossiche per il singolo paziente, perché tagliate su misura (genomica, epigenetica, ambientale) o meglio ancora sulla correlazione dei diversi strati dell'h-GIS, ma a condurre l'approccio terapeutico è largamente la diagnostica. Per spiegare la MP Francis Collins [8] fa riferimento alle possibilità non remote che la conta dei globuli bianchi sia sostituita dall'individuazione di centinaia di tipi cellulari diversi sotto il profilo immunologico, che sensori e connettività consentano monitoraggi continui e real-time di glicemia, pressione arteriosa e ritmo cardiaco, che la genotipizzazione individui fattori protettivi verso specifiche malattie, che da campioni fecali si possa risalire a pattern di microbioma responsabili dell'obesità, o che la precoce identificazione di cellule e di acidi nucleici tumorali nel torrente circolatorio consenta una precoce rilevazione del cancro o delle sue ricadute (biopsia liquida). Alcuni autori [22] prevedono, addirittura, uno shift di fondi verso la diagnostica, perché individuare il corrispettivo diagnostico, specificamente molecolare e genetico, dei trattamenti è fondamentale, anche se ricordano che è l'integrazione dei fattori genetici, epigenetici, ambientali e di vita che caratterizza la MP.

Sono i biomarcatori a svolgere un ruolo chiave nello "sblocco" della MP [30] e questo ha a che fare con la Medicina Traslazionale (MT) [31] e con la sua inter-relazione con la Medicina di Laboratorio (ML) [12]. Il processo di identificare e validare un test per biomarcatori e sviluppare e valutare una terapia targeted è assai complesso [12]. In particolare per la fase diagnostica è necessario definire la validità analitica del marcatore, cioè che sia accurato e affidabile, la sua validità clinica, che sia correlato a una malattia e a un esito di interesse clinico, e la sua utilità clinica, che porti effettivamente a un miglioramento dell'outcome del paziente in confronto con i trattamenti standard. Il processo inizia nei Laboratori di ricerca per la definizione delle caratteristiche del test e la sua efficacia come target/indicatore di target e poi si sposta nei Laboratori di Medicina di Laboratorio per le valutazioni popolazionistiche del test, mentre corrispettivi studi di fasi successive (I, II, III) valutano l'effectiveness e la sicurezza della terapia correlata. La "qualità" del marcatore è pertanto essenziale [32] e richiede "qualità" metodologica [33], una comprensione corretta del rapporto tra innovazione ed "evidenze" che caratterizza MT [31], e nuovi ruoli per
ML [15]. La prima esigenza è così sentita che recentemente l'Institute of Medicine (IOM) ha costituito un Committee on Policy Issues in the Clinical Development and Use of Biomarkers for Molecularly Targeted Therapies per studiare gli aspetti più importanti dello sviluppo dei biomarcatori e delle terapie correlate, il quale nel marzo 2016 ha rilasciato 10 raccomandazioni [30] che toccano molti punti salienti: standard di utilità clinica, processi di revisione (FDA e altri), trasparenza, standard di accreditamento, intraoperabilità digitale, un repository nazionale facilmente consultabile, indicazioni per il superamento delle barriere, standard per il campionamento, standard per le linee guida. Il flusso della traslazione [12] attraversa passaggi che vedono all'opera figure professionali diverse, tra le quali ML assume un valore chiave per le sue caratteristiche di ponte tra il versante "scientifico" e quello "pratico" della Medicina. Un ruolo stimolante di generazione, verifica e diffusione delle evidenze coinvolge il patologo clinico, in particolare con la disponibilità di big data popolazionistici [34]. Il patologo è sfidato dalla nuova diagnostica di precisione e, seppure dal versante anatomo-patologico ma facilmente trasferibile a quello clinico-patologico (basti pensare alle "biopsie liquide"), si è auspicata [35] un'estensione del suo ruolo come integratore di dati per diagnosticare e classificare malattie, come modellatore di processi fisiopatologici e diagnostici e come suggeritore di raccomandazioni terapeutiche correlate. La Patologia Terapeutica, d'altra parte, è solo una delle nuove aperture di senso che Brain Smith indica alla comunità professionale di ML di fronte all'innovazione radicale (o distruttiva) definita da "omiche", miniaturizzazione, iperconnettività (digitale e mobile) e big data [31].

Conflitti di interesse Nessuno.

Studi condotti su esseri umani e animali Non applicabile.

Consenso informato Non applicabile.

\section{Bibliografia}

1. FACT SHEET: President Obama's Precision Medicine Initiative (2015) The White House Web site. http://1.usa.gov/1T7b7m5 (Accesso 2 settembre 2016)

2. Boguski MS, Arnaout R, Hill C (2009) Customized care 2020: how medical sequencing and network biology will enable personalized medicine. F1000 Biol Rep 1:73. http://f1000.com/prime/ reports/10.3410/B1-73 (Accesso 2 settembre 2016)

3. https://pm.catapult.org.uk (Accesso 2 settembre 2016)

4. Roberts S, Jiulius M (2016) Precision medicine: now, not when. Healthc Manag Forum 29:158-161

5. http://wisesociety.it/ambiente-e-scienza/human-technopole-italia2040-nasce-il-dopo-expo/ (Accesso 2 settembre 2016)

6. https://www.nih.gov/precision-medicine-initiative-cohortprogram (Accesso 2 settembre 2016)

7. http://www8.nationalacademies.org/onpinews/newsitem.aspx? recordid=13284 (Accesso 2 settembre 2016) 
8. Collins FS, Varmus H (2015) A new iniziative on precision medicine. N Engl J Med 372:793-795

9. Roden DM, Tyndale RF (2013) Genomic medicine, precision medicine, personalized medicine: what's in a name? Clin Pharmacol Ther 94:169-172

10. http://www.plengegen.com/wp-content/uploads/4_TowardPrecision-Medicine.pdf (Accesso 2 settembre 2016)

11. Schleidgen S, Klingler C, Bertram T et al (2013) What is personalized medicine: sharpening a vague term based on a systematic literature review. BMC Med Ethics 14:55-66

12. Cappelletti P (2009) La Medicina Personalizzata fra ricerca e pratica clinica: il ruolo della Medicina di Laboratorio. RIMeL/IJLaM 5(Suppl.):26-32

13. http://www.nature.com/nm/journal/v19/n3/full/nm0313-249.html (Accesso 2 settembre 2016)

14. Cappelletti P (2016) Il paziente autonomo. Riv Ital Med Lab 12:62-64

15. Cappelletti P (2014) La complessità e la Medicina di Laboratorio. Riv Ital Med Lab 10:187-192

16. De Grandis G, Halgunset V (2016) Conceptual and terminological confusion around personalised medicine: a coping strategy. BMC Med Ethics 17:43-54

17. http://www.cancer.gov/news-events/cancer-currents-blog/2015/ precision-medicine-initiative-2016 (Accesso 2 settembre 2016)

18. Cappelletti P (2015) Il mutamento delle professioni della Medicina di Laboratorio. Riv Ital Med Lab 11:123-131

19. http://oxfordmedicine.com/view/10.1093/med/9780199934959. 001.0001/med-9780199934959-chapter-83 (Accesso 2 settembre 2016)

20. Galli SJ (2016) Toward precision medicine and health: opportunities and challenges in allergic diseases. J Allergy Clin Immunol 137:1289-1300

21. Jameson JL, Longo DL (2015) Precision medicine-personalized, problematic, and promising. N Engl J Med 372:2229-2234
22. http://www.journalofclinicalpathways.com/article/potentials-andbarriers-precision-medicine-oncology (Accesso 2 settembre 2016)

23. http://forward.recentiprogressi.it/wp-content/uploads/2016/01/ FWD-01web.pdf (Accesso 2 settembre 2016)

24. Stanek EJ, Sanders CL, Taber KA et al (2012) Adoption of pharmacogenomic testing by US physicians: results of a nationwide survey. Clin Pharmacol Ther 91:450-458

25. Vassy JL, Korf BR, Green RC (2015) How to know when physicians are ready for genomic medicine. Sci Transl Med 7:287fs 19

26. http://www.journalofclinicalpathways.com/blog/precisionmedicine-it-realistic (Accesso 2 settembre 2016)

27. Bayer R, Galea S (2015) Public health in the precision medicine era. N Engl J Med 373:499-501

28. https://www.edifecs.com/e/the-4-ps-of-precision-medicine/ (Accesso 2 settembre 2016)

29. http://www.himss.org/library/interoperability-standards/what-isinteroperability (Accesso 2 settembre 2016)

30. Lyman GH, Moses HL (2016) Biomarker tests for molecularly targeted therapies - the key to unlocking precision medicine. N Engl J Med 375:4-6

31. Cappelletti P (2013) Innovazione ed evidenze in Medicina di Laboratorio. Riv Ital Med Lab 9:177-184

32. Hayes DF, Allen J, Compton C et al (2013) Breaking a vicious cycle. Sci Transl Med 5:196cm6

33. Cappelletti P (2003) La Medicina Molecolare e la Medicina di Laboratorio. Riv Med Lab/JLM 4(Suppl.):19-31

34. Dorizzi RM, Baldrati L, Vascotto L et al (2012) Scenari e ruoli della Medicina di Laboratorio al tempo della crisi: governance clinica, EBLM, linee guida, PDT/PA. Riv Ital Med Lab $8: 216-230$

35. Walk EE (2009) The role of pathologists in the era of personalized medicine. Arch Pathol Lab Med 133:605-610 